\title{
The Preoperative Neutrophil Lymphocyte Ratio and Platelet Lymphocyte Ratio Predicts Disease-Free Survival in Resectable Esophageal Squamous Cell Carcinoma
}

\author{
Zhiwei Zheng ${ }^{l, *}$ \\ Cui Yang ${ }^{2, *}$ \\ Chun Cai ${ }^{\prime}$ \\ Huide Zhu' \\ 'Department of Pharmacy, Cancer \\ Hospital of Shantou University Medical \\ College, Shantou, 5I504I, People's \\ Republic of China; ${ }^{2}$ Department of \\ Obstetrics and Gynecology Shantou \\ Central Hospital, Shantou, 5I5000, \\ People's Republic of China \\ *These authors contributed equally to \\ this work
}

Background: Recent studies have revealed that the presence of systemic inflammation is associated with poor survival for esophageal squamous cell carcinoma. We aimed to investigate prognostic values of preoperative neutrophil lymphocyte ratio (NLR) and platelet lymphocyte ratio (PLR) in resectable esophageal squamous cell carcinoma.

Methods: A cohort of 167 resectable ESCC patients was retrospectively reviewed between January 2017 and September 2020. The best cut-off value of NLR and PLR was selected by plotting the receiver operating characteristic curve. All reviewed patients were divided into high NLR/PLR or low NLR/PLR group to evaluate prognostic factors.

Results: Among the 167 patients, 34 (20.36\%) were women and 133 (79.64\%) were men. The mean age was $62.64 \pm 7.91$ years, with an age range from 44 to 85 years. All patients were divided into low NLR $(<2.20)$ or high NLR $(\geq 2.20)$ group (AUC $=62.5 \%$ with the sensitivity of $61.8 \%$ and specificity $60.9 \%, \mathrm{P}=0.025)$, low PLR $(<110)$ or high PLR $(\geq 110)$ group ( $\mathrm{AUC}=59.6 \%$ with the sensitivity of $82.4 \%$ and specificity $35.3 \%, \mathrm{P}=0.083$ ). High NLR and PLR were associated with a larger tumor diameter $(\mathrm{P}<0.05)$, while high NLR was also associated with poor tumor classification $(\mathrm{P}=0.022)$. There was a positive correlation between NLR and PLR $(r=0.614, \mathrm{P}<0.001)$. High NLR and PLR were significantly associated with poor disease-free survival. Multivariate analyses identified NLR as a prognostic factor in resectable ESCC.

Conclusion: The NLR and PLR predict disease-free survival in resectable esophageal squamous cell carcinoma.

Keywords: neutrophil lymphocyte ratio, platelet lymphocyte ratio, disease-free survival, resectable esophageal squamous cell carcinoma

\section{Introduction}

Esophageal cancer (EC) is one of the most common malignant tumors worldwide, the crude mortality rate of EC in 2020 was $7.8 / 100,000$, which represented $5.5 \%$ of all cancer deaths and ranked as the sixth most common cause of cancer death. ${ }^{1}$ Esophageal squamous cell carcinoma (ESCC) is a major type of EC. Despite advances in multidisciplinary treatment of ESCC, surgical resection remains the modality of choice, while the survival after surgical resection remains poor. ${ }^{2}$ Therefore, it will become more and more important to find prognostic factors for ESCC. In fact, several clinicopathological factors, such as tumor differentiation and
Correspondence: Huide Zhu Department of Pharmacy, Cancer Hospital of Shantou University Medical

College, Shantou, 5I504I, People's Republic of China

Tel $+86075488555844-$ I I 40

Fax +86075488555844

Email zhuhuide_2009@163.com 
TNM staging, have been widely used to predict prognosis in patients with ESCC. However, even among patients with the same clinicopathological factors, clinical outcomes vary widely. Therefore, a new accurate prognosis biomarker for ESCC patients is needed to identify highrisk patients with poor prognosis.

Recently, there is increasing evidence that systemic inflammatory responses have prognostic value in a variety of cancers. Indeed, the inflammatory biomarkers, including the neutrophil to lymphocyte ratio (NLR), platelet to lymphocyte ratio (PLR), have been reported to be significantly associated with the prognosis of the cancers. ${ }^{3}$ However, there is still controversy that which inflammatory biomarker is the best predictor of ESCC prognosis. Until now, few studies have focused on the association between inflammatory biomarkers and predicts diseasefree survival in resectable esophageal squamous cell carcinoma. In addition, there is still debate about the optimal cut-off values for these inflammatory biomarkers in predicting prognosis. Therefore, the purpose of this study was to explore the clinical significance of NLR and PLR as independent prognostic factors in patients with resectable ESCC and to identify which is the more effective in this role.

\section{Methods}

\section{Patients Selection}

A retrospective analysis was performed on data from 167 patients with ESCC that underwent radical surgery at cancer hospital of Shantou university medical college (Guangdong, China) between January 2017 and September 2020. All of the patients included in the analysis fit the criteria: (1) ESCC confirmed by histopathology; (2) surgery or preceded by adjuvant chemotherapy/radiotherapy before surgery; (3) curative esophagectomy with $\mathrm{R} 0$ resection (en bloc resection with margins histologically free of disease); (4) preoperative NLR and PLR obtained before esophagectomy. Patients who underwent palliative surgery and clinical evidence of infection, systemic inflammation or autoimmune disorder, were excluded.

\section{Data Collection and Definitions}

Patient clinical information, including age, sex and operation date, clinicopathological features and preoperative laboratory data were collected from the medical records. Telephone interviews were used to follow-up each patient. The last follow-up date was September 2020. Disease-free survival (DFS) time defined as the time between surgery and cancer metastasis/dead from any cause or to the last date of follow-up.

\section{NLR and PLR}

The neutrophil, lymphocyte and platelet counts were collected using a routine blood test within one week before surgery. NLR was defined as the absolute neutrophil count divided by the absolute lymphocyte count. PLR was defined as the absolute platelet count divided by the absolute lymphocyte count.

\section{Statistical Analysis}

All data were analyzed using IBM SPSS software version 21. By drawing the receiver operating characteristic curve (ROC) of the preoperative NLR and PLR levels of the patients, Youden index (sensitivity + specificity -1 ) and area under the curve (AUC) were calculated. The optimal preoperative cut-off vale of NLR and PLR were determined, on which the continuous variables were converted to categorical variables.

Continuous variables are presented as the mean \pm standard error and categorical variables are presented as frequencies and percentages. Independent sample $t$-test or one-way ANOVA was used for comparison of measurement data, and chi-square test was used for comparison of enumeration data. Disease-free survival rate was calculated using the Kaplan-Meier method of univariate analysis, and the differences were compared using the Log rank test. Independent prognostic factors were carried out by Cox regression model of multivariate analysis. Each test was two-tailed. $\mathrm{P}<0.05$ was considered to indicate a statistically significant difference.

\section{Results}

\section{The ROC Curve for Optimal Cutoff Value and AUC}

The ROC curve are plotting as Figure 1. When the NLR was 2.20, YI index was at its maximum ( $\mathrm{YI}=0.234$ ), demonstrating that 2.20 was the optimal cutoff value for NLR (AUC $=62.5 \%$ with the sensitivity of $61.8 \%$ and specificity $60.9 \%, \mathrm{P}=0.025)$. Therefore, patients were divided into low NLR $(<2.20)$ and high NLR $(\geq 2.20)$ groups. Similarly, When the PLR was 110, YI was at its maximum ( $\mathrm{YI}=0.177$ ), demonstrating that 110 was the optimal cutoff value for PLR (AUC $=59.6 \%$ with the sensitivity of $82.4 \%$ and a specificity $35.3 \%, \quad \mathrm{P}=0.083$ ). 

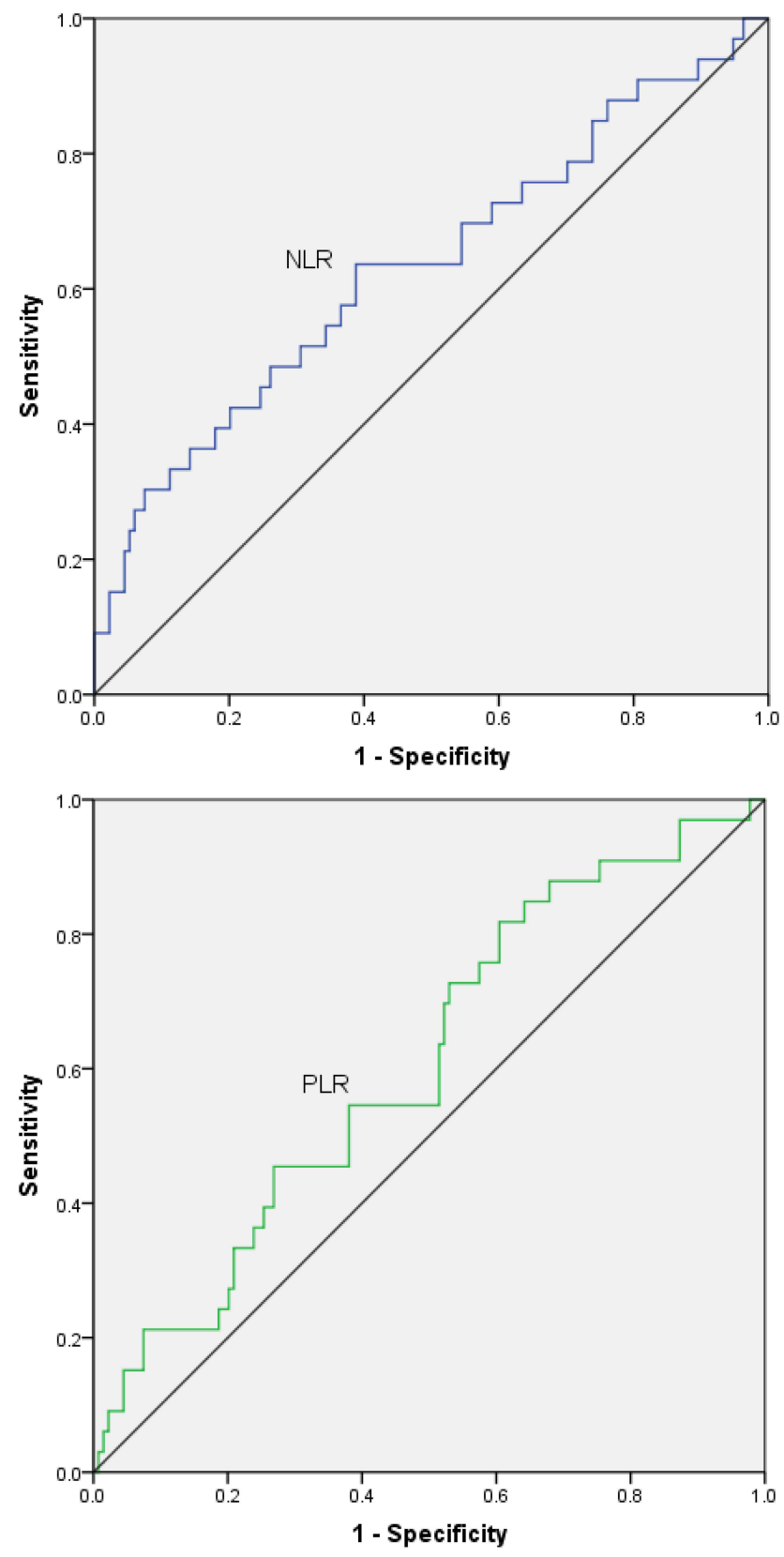

Figure I ROC curves for NLR and PLR. The ROC curve for NLR is indicated by the blue line. The ROC curve for PLR is indicated by the green line.

Therefore, patients were divided into low PLR $(<110)$ and high PLR $(\geq 110)$ groups.

\section{Patient Characteristics}

Among the 167 patients, 34 (20.36\%) were women and $133(79.64 \%)$ were men. The mean age was $62.64 \pm 7.91$ years, with an age range from 44 to 85 years. All the clinicopathologic characteristics were comparable between patients grouped by NLR or PLR, as shown in Table 1 . Our study showed that NLR was associated with tumor size, T classification. However, PLR was found associated with tumor size only. High NLR and PLR were associated with a larger tumor diameter $(\mathrm{P}<0.05)$. A high NLR was also associated with a poor primary tumor classification $(\mathrm{P}=0.022)$. In addition, there was a positive correlation between the NLR and PLR $(r=0.614, \mathrm{P}<0.001)$ (Figure 2).

Association of NLR and PLR with the disease-free survival (DFS). In total, 167 patients were enrolled in the present study. The median follow-up time was 363 days, with a range of 29-1238 days. At the final follow-up date, 163 patients $(97.64 \%)$ were alive. Tumor metastasis occurred in 34 patients during follow-up. Patients with a high NLR possessed a significantly poorer disease-free survival compared with patients with low NLR ( $\mathrm{P}=0.013$; Figure 3). Patients with high PLR possessed significantly poorer disease-free survival compared with patients with low PLR ( $\mathrm{P}=0.083$; Figure 4).

Multivariate analyses of independent prognostic factors. Multivariate analyses using the Cox proportional hazards model identified NLR ( $\mathrm{P}=0.032$; 95\% CI, $1.033-$ 2.093) as independent prognostic factors (Table 2). The risk of succumbing to resectable esophageal squamous cell carcinoma for patients with a high NLR was 1.47 times higher than patients with a low NLR $(\mathrm{P}=0.032)$.

\section{Discussion}

Over the past decades, it has become widely accepted that cancer and inflammation are linked. ${ }^{4,5}$ Accumulating studies have demonstrated that systemic inflammatory response, particularly NLR, and PLR, could be an independent prognostic predictor in a variety of carcinomas including ESCC. ${ }^{6}$ A retrospective study with 65 patients following esophageal resection proved that a higher NLR predicts complications. ${ }^{7}$ Indeed, regardless of stage, the maintenance and development of the systemic inflammatory responses have been consistently linked to poorer prognosis. ${ }^{8,9}$ To the best of our knowledge, in patients with resectable esophageal squamous cell carcinoma, up to now, a potential prognostic value has not been investigated. Therefore, we evaluated the prognostic significance of NLR and PLR regarding two different endpoints.

Our study showed that high NLR and PLR were significantly associated with larger tumor diameter, while high NLR were also significantly associated with a poor primary tumor Classification. In addition, the results of the Kaplan-Meier analysis showed that these two inflammatory biomarkers were significantly associated with poorer 
Table I Clinicopathological Characteristics of 167 Patients, Grouped by NLR and PLR

\begin{tabular}{|c|c|c|c|c|c|c|}
\hline \multirow[t]{2}{*}{ Characteristics } & \multicolumn{3}{|c|}{ NLR } & \multicolumn{3}{|c|}{ PLR } \\
\hline & $\begin{array}{l}\text { NLR<2.20 (Number, } \\
\%)\end{array}$ & $\begin{array}{l}\text { NLR } \geq 2.20 \\
\text { (Number, \%) }\end{array}$ & P-value & $\begin{array}{l}\text { PLR<I I } 0 \\
\text { (Number, \%) }\end{array}$ & $\begin{array}{l}\text { PLR } \geq \mathrm{I} I 0 \\
\text { (Number, \%) }\end{array}$ & P-value \\
\hline Total Gender & $93(100)$ & $74(100)$ & & $53(100)$ & $114(100)$ & \\
\hline Male & $71(76.3)$ & 62(83.78) & 0.236 & $42(79.25)$ & $91(79.82)$ & 0.931 \\
\hline Female & $22(23.66)$ & $12(16.22)$ & & II(20.75) & $23(20.18)$ & \\
\hline Age (years) & $62.60 \pm 7.77$ & $62.69 \pm 8.14$ & 0.944 & $62.98 \pm 7.74$ & $62.48 \pm 8.02$ & 0.706 \\
\hline $\begin{array}{l}\text { Tumor } \\
\text { diameter }(\mathrm{cm})\end{array}$ & $3.86 \pm 1.33$ & $4.67 \pm 1.87$ & 0.002 & $3.80 \pm 1.23$ & $4.40 \pm 1.76$ & 0.013 \\
\hline $\begin{array}{l}\text { Tumor location } \\
\text { Upper } \\
\text { Middle } \\
\text { Lower }\end{array}$ & $\begin{array}{l}10(10.75) \\
56(60.22) \\
27(29.03)\end{array}$ & $\begin{array}{l}13(17.57) \\
37(50.00) \\
24(32.43)\end{array}$ & 0.314 & $\begin{array}{l}6(11.32) \\
30(56.60) \\
17(32.08)\end{array}$ & $\begin{array}{l}17(14.91) \\
63(55.26) \\
34(29.83)\end{array}$ & 0.814 \\
\hline $\begin{array}{l}\text { Differentiation } \\
\text { Well } \\
\text { Moderate } \\
\text { Poor }\end{array}$ & $\begin{array}{c}7(7.53) \\
7 I(76.34) \\
15(16.13)\end{array}$ & $\begin{array}{c}3(4.05) \\
57(77.03) \\
14(18.92)\end{array}$ & 0.601 & $\begin{array}{c}4(7.55) \\
36(67.92) \\
13(24.53)\end{array}$ & $\begin{array}{c}6(5.26) \\
92(80.70) \\
16(14.04)\end{array}$ & 0.185 \\
\hline $\begin{array}{l}\text { T classification } \\
\text { T1 } \\
\text { T2 } \\
\text { T3 } \\
\text { T4 }\end{array}$ & $\begin{array}{c}26(27.96) \\
17(18.28) \\
46(49.46) \\
4(4.3)\end{array}$ & $\begin{array}{c}9(12.16) \\
9(12.16) \\
49(66.22) \\
7(9.46)\end{array}$ & 0.022 & $\begin{array}{c}14(26.42) \\
9(16.98) \\
27(50.94) \\
3(5.66)\end{array}$ & $\begin{array}{l}21(18.42) \\
17(14.91) \\
68(59.65) \\
8(7.02)\end{array}$ & 0.618 \\
\hline $\begin{array}{l}\text { Nodal metastasis } \\
\text { Negative } \\
\text { Positive }\end{array}$ & $\begin{array}{l}48(51.61) \\
45(48.39)\end{array}$ & $\begin{array}{l}32(43.24) \\
42(56.76)\end{array}$ & 0.282 & $\begin{array}{l}26(49.06) \\
27(50.94)\end{array}$ & $\begin{array}{l}54(47.37) \\
60(52.63)\end{array}$ & 0.839 \\
\hline
\end{tabular}

disease-free survival. However, until now, the mechanism and relationship between inflammatory biomarkers and ESCC prognosis remains unclear. Accumulating studies

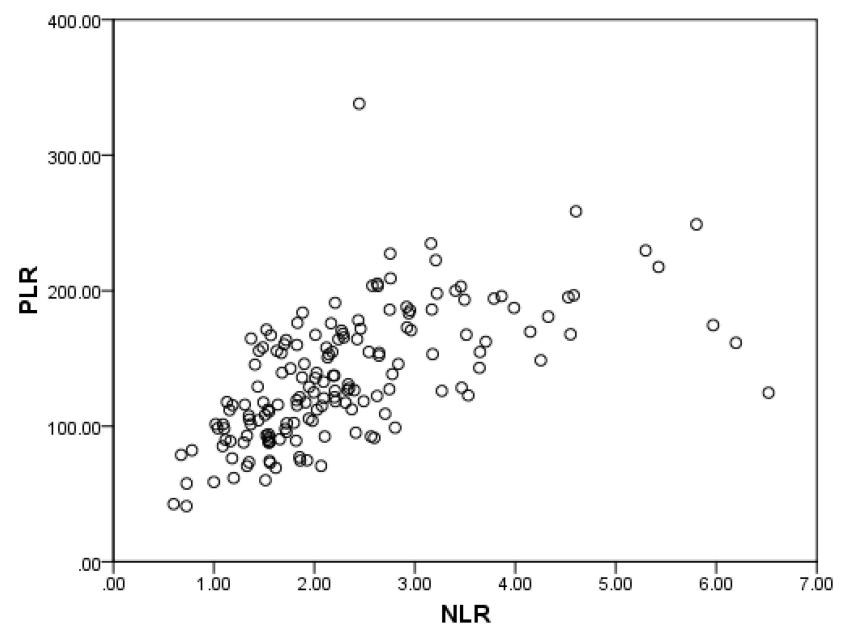

Figure 2 Correlation between the NLR and PLR $(r=0.614, P<0.001)$. Abbreviations: NLR, neutrophil lymphocyte ratio; PLR, platelet lymphocyte ratio. have demonstrated that systemic inflammatory response, particularly NLR and PLR could be an independent prognostic predictor in a variety of carcinomas including ESCC. ${ }^{6,10}$ It has been reported that neutrophils and platelets promote cancer development through different mechanisms, while lymphocytes are needed to eliminate tumor cells. Thus, this may partially explain why high rates of NLR and PLR are significantly associated with poor prognosis in ESCC patients.

To date, there is no consensus on which inflammatory biomarkers are most useful clinically and which are the best predictors of prognosis in the study. ${ }^{11}$ In our study, we compared NLR and PLR using their respective AUC values. According to the present results, the AUC for NLR was 0.625 , which was greater compared with PLR (AUC $=0.5960$ ). The present results demonstrated that the risk of patients succumbing to ESCC was increased in high NLR compared with high PLR (RR, 1.470 vs.0.997). The AUC and RR data indicate that NLR is 


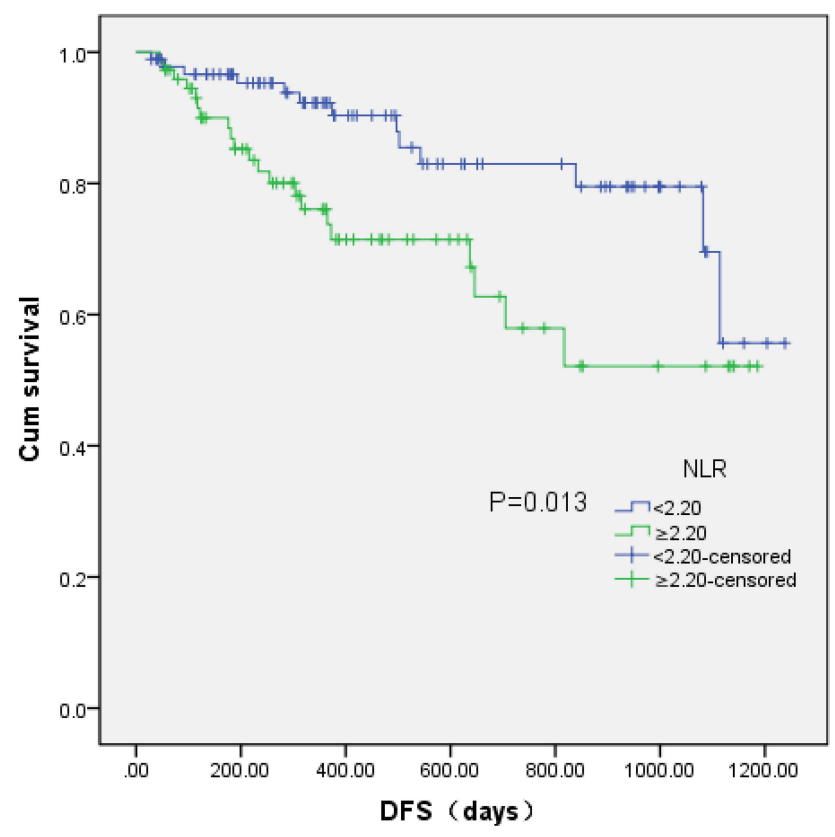

Figure 3 DFS curve grouped by NLR. Patients with high NLR $(\geq 2.20)$ possessed significantly poorer DFS time compared with patients with low NLR $(<2.20 ; P=0.013)$. Abbreviations: DFS, disease-free survival; NLR, neutrophil lymphocyte ratio; Cum., cumulative.

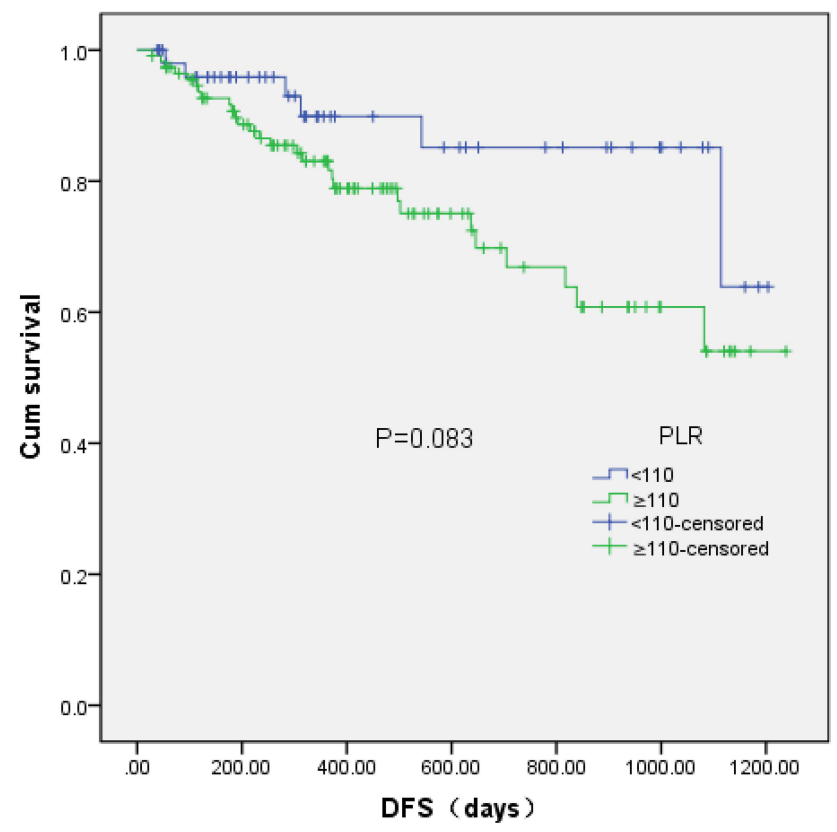

Figure 4 DFS curve grouped by PLR. Patients with high PLR $(\geq 110)$ possessed significantly poorer DFS time compared with patients with low PLR $(<110 ; P=0.083)$. Abbreviations: DFS, disease-free survival; PLR, platelet-to-lymphocyte ratio; Cum., cumulative.

superior to PLR as a predictive factor for patients with ESCC.

But our study has several limitations. First of all, it is a retrospective study including only one institution, which
Table 2 Multivariate Analyses in 167 Patients with Resectable Esophageal Squamous Cell Carcinoma

\begin{tabular}{|l|l|l|}
\hline Features & P-value & RR (95\% CI) \\
\hline NLR & 0.032 & $I .470(I .033-2.093)$ \\
PLR & 0.522 & $0.997(0.987-I .007)$ \\
Tumor diameter & 0.245 & $I .122(0.9241 .353)$ \\
T classification & 0.139 & $I .456(0.886-2.394)$ \\
\hline
\end{tabular}

Abbreviations: $\mathrm{RR}$, relative risk; $\mathrm{Cl}$, confidence interval; NLR, neutrophil lymphocyte ratio; PLR, platelet to lymphocyte ratio.

may lead to a selection bias. Second, with limited data availability, we did not study other inflammatory biomarkers, such as C-reactive protein and the lymphocyte to monocyte ratio. Third, due to the follow-up time, we lack the 5-year survival rate, and our conclusions may be strengthened by using other survival measures. The long data collection time in this retrospective analysis and advances in surgical technology during this period may influence the clinical outcome.

In conclusion, the present study validates the use of preoperative NLR and PLR as independent disease-free survival prognostic factors for resectable esophageal squamous cell carcinoma patients. Notably, NLR was observed to be more effective than PLR for predicting ESCC outcome. However, as an observational, single-hospital, small-scale study, the present study is limited. Larger prospective studies are required to confirm these preliminary results.

\section{Conclusion}

The NLR and PLR predict disease-free survival in resectable esophageal squamous cell carcinoma in our study, large-scale prospective research are needed to prove our findings. It may have important clinical utility for identifying patients at high risk and guiding the administration of individualized treatment.

\section{Ethics}

The study was reviewed and approved by the Ethics Committee of the Cancer Hospital of Shantou University Medical College. The requirement for informed consent was waived because the medical records used in this study were obtained from previous clinical treatments and it will not adversely affect the rights and health of the subject. We declare that we would protect the confidentiality of personal information of research subjects. This research was conducted in accordance with the Declaration of Helsinki. 


\section{Acknowledgments}

This work was supported by Science and Technology Special Fund of Guangdong Province of China (190829105556145).

\section{Disclosure}

The authors report no conflicts of interest in this work.

\section{References}

1. Sung H, Ferlay J, Siegel RL, et al. Global cancer statistics 2020: GLOBOCAN estimates of incidence and mortality worldwide for 36 cancers in 185 countries. CA Cancer J Clin. 2021;71:209-249. doi: $10.3322 /$ caac. 21660

2. Ali Mohammad FH, Go P, Ghanem T, Stachler R, Hammoud Z. Longterm survival after local resection of cervical esophageal cancer. Ann Thorac Surg. 2015;99(6):2202-2203. doi:10.1016/j.athoracsur.2014. 08.050

3. Cupp MA, Cariolou M, Tzoulaki I, Aune D, Evangelou E, BerlangaTaylor AJ. Neutrophil to lymphocyte ratio and cancer prognosis: an umbrella review of systematic reviews and meta-analyses of observational studies. BMC Med. 2020;18(1):360. doi:10.1186/s12916-02001817-1

4. Singh N, Baby D, Rajguru JP, Patil PB, Thakkannavar SS, Pujari VB. Inflammation and cancer. Ann Afr Med. 2019;18(3):121-126. doi:10.4103/aam.aam_56_18
5. Kay J, Thadhani E, Samson L, Engelward B. Inflammation-induced DNA damage, mutations and cancer. DNA Repair (Amst). 2019;83: 102673.

6. Song Q, Wu JZ, Jiang HF, Wang S, Cai SN. The postoperative lymphocyte to monocyte ratio change predicts poor clinical outcome in patients with esophageal squamous cell carcinoma undergoing curative resection. Dis Markers. 2020;2020:1451864. doi:10.1155/ 2020/1451864

7. Vulliamy P, McCluney S, Mukherjee S, Ashby L, Amalesh T. Postoperative elevation of the neutrophil: lymphocyte ratio predicts complications following esophageal resection. World J Surg. 2016;40 (6):1397-1403. doi:10.1007/s00268-016-3427-z

8. Zhang N, Jiang J, Tang S, Sun G. Predictive value of neutrophil-lymphocyte ratio and platelet-lymphocyte ratio in non-small cell lung cancer patients treated with immune checkpoint inhibitors: a meta-analysis. Int Immunopharmacol. 2020;85:106677. doi:10.1016/j.intimp.2020.106677

9. HanNing T, BiHui $\mathrm{P}, \mathrm{Li} \mathrm{W}$, et al. C-reactive protein-to-albumin ratio is an independent poor prognostic factor in newly diagnosed chronic lymphocytic leukaemia: a clinical analysis of 322 cases. Transl Oncol. 2021;14(4):101035.

10. Song Q, Wu JZ, Wang S. Low preoperative lymphocyte to monocyte ratio serves as a worse prognostic marker in patients with esophageal squamous cell carcinoma undergoing curative tumor resection. J Cancer. 2019;10(9):2057-2062. doi:10.7150/jca.29383

11. Gayaf M, Karadeniz G, Güldaval F, Polat G, Türk M. Which one is superior in predicting 30 and 90 days mortality after COPD exacerbation: DECAF, CURB-65, PSI, BAP-65, PLR, NLR. Expert Rev Respir Med. 2021;1-7. doi:10.1080/17476348.2021.1901584

\section{Publish your work in this journal}

Cancer Management and Research is an international, peer-reviewed open access journal focusing on cancer research and the optimal use of preventative and integrated treatment interventions to achieve improved outcomes, enhanced survival and quality of life for the cancer patient.
The manuscript management system is completely online and includes a very quick and fair peer-review system, which is all easy to use. Visit http://www.dovepress.com/testimonials.php to read real quotes from published authors. 